\title{
An Update on the CHARA Array
}

\author{
T.A. ten Brummelaar ${ }^{\text {la }}$, D.G. Gies ${ }^{\mathrm{b}}$, H.A. McAlister ${ }^{\mathrm{b}}$, S.T. Ridgway ${ }^{\mathrm{c}}$, J. Sturmann ${ }^{\mathrm{a}}$, L. Sturmann ${ }^{\mathrm{a}}$, \\ G.H. Schaefer ${ }^{\mathrm{a}}$, N.H. Turner ${ }^{\mathrm{a}}$, C.D. Farrington ${ }^{\mathrm{a}}$, N.J. Scott ${ }^{\mathrm{c}}$, J.D. Monnier ${ }^{\mathrm{d}}$, and M.J. Ireland ${ }^{\mathrm{e}}$. \\ ${ }^{a}$ The CHARA Array, Georgia State University, Mount Wilson CA, USA 91012; ${ }^{b}$ Center for High \\ Angular Resolution Astronomy, Georgia State University, PO Box 4106, Atlanta GA, 30302-4106 \\ USA; 'NASA Ames Research Center, Moffett Field, CA 94035, USA; 'University of Michigan, \\ Astronomy Department, Ann Arbor, MI 48109-1107, USA; ${ }^{\mathrm{e}}$ Australian National University, \\ Research School of Astronomy \& Astrophysics, Mount Stromlo Observatory, Weston Creek, ACT \\ 2611, AUSTRALIA
}

\begin{abstract}
The CHARA Array, operated by Georgia State University, is located at Mount Wilson Observatory just north of Los Angeles in California. The CHARA consortium includes many groups, including LIESA in Paris, Observatoire de la Cote d'Azur, the University of Michigan, Sydney University, the Australian National University, the NASA Exoplanet Science Institute, and most recently the University of Exeter. The CHARA Array is a six-element optical/NIR interferometer, and for the time being at least, has the largest operational baselines in the world. In this paper we will give a brief introduction to the array infrastructure with a focus on our Adaptive Optics program, and then discuss current funding as well as opportunities of funding in the near future.
\end{abstract}

Keywords: Optical ground based interferometry, Center for High Angular Resolution Astronomy

\section{INTRODUCTION}

In this paper we continue a lengthy series of updates of the CHARA Array at these meetings ${ }^{1,2,3,4,5,6 \& 7}$. In this update we will very briefly describe the CHARA Array infrastructure, and then spend more time on the most significant upgrade the CHARA Array Adaptive Optics Program. We will then briefly describe the current status of funding at CHARA and the prospects for new funding in the near future.

\section{OVERVIEW OF THE CHARA ARRAY}

The CHARA Array's six 1-m aperture telescopes are arranged in a Y-shaped configuration yielding 15 interferometric baselines from 33 to 331 meters as well as 10 independent closure phases. These include the longest OIR baselines yet implemented anywhere in the world and permit resolutions at the sub-mas level. The facility's primary components and sub-systems, shown in Figure 1, are described more fully by ten Brummelaar et al. ${ }^{8}$ but will be briefly described here.

\subsection{Light Collecting Telescopes}

Each of the six telescopes injects a $12.5-\mathrm{cm}$ collimated beam into the vacuum transport tubes. The secondary is actively tip/tilt controlled. The custom-designed mounts are exceptionally stiff and massive $(23,000 \mathrm{lbs})$ and designed for long term focus stability. The telescope enclosures are comprised of two concentric cylinders, each of which can be raised and lowered to provide high-throughput passive ventilation. The final mirror in the telescope optical train injects the collimated $12.5-\mathrm{cm}$ beam through an optical window into a vacuum tube system (at $\sim 1$ Torr) for relay to the central beam combination facility.

\subsection{Optical Path Length Compensation}

Maintenance of zero optical path length difference is accomplished in two stages. The first occurs in vacuum and employs six parallel systems dubbed the "Pipes of Pan" (POPs). Remotely actuated mechanisms move mirrors to select appropriate static delay segments. Upon exiting the PoPs, the beams leave vacuum and are injected into the continuous

1. theo@chara-array.org Phone: 6267968607

Optical and Infrared Interferometry and Imaging V, edited by Fabien Malbet,

Michelle J. Creech-Eakman, Peter G. Tuthill, Proc. of SPIE Vol. 9907, 990703

(c) 2016 SPIE · CCC code: 0277-786X/16/\$18 - doi: 10.1117/12.2232125 
delay. The "Optical Path Length Equalizers" (OPLEs) utilize cats-eye retro-reflectors on steel rail pairs $46 \mathrm{~m}$ long and are controlled by a four-tiered servo system utilizing laser metrology feedback to achieve positioning to $\pm 10 \mathrm{~nm}$ r.m.s.

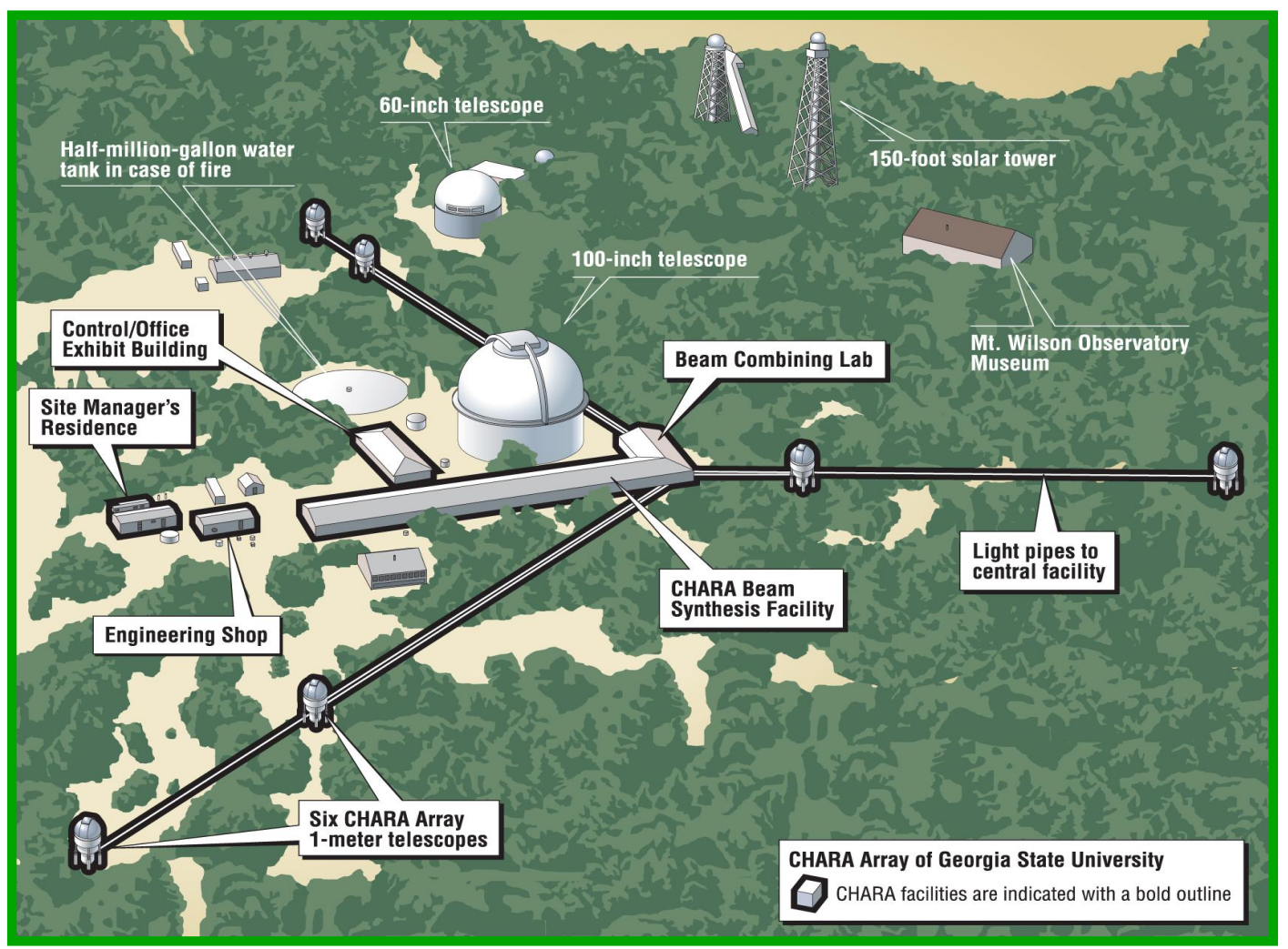

Figure 1. A cartoon of the layout of the CHARA Array showing all six telescope positions and major facilities.

\subsection{Beam Management}

The beams emerging from the OPLEs are reduced to a final diameter of $1.9 \mathrm{~cm}$ by two-element "beam-reducing telescopes." They then pass through the laboratory AO system described below and then through a "longitudinal dispersion corrector" that compensates for differential dispersion caused by unequal air paths. Finally, the "beam sampling system" (BSS) separates visible from IR light at the $1-\mu \mathrm{m}$ boundary and sends the light to the visible and IR beam combiners.

\subsection{Beam Combination}

Through formal agreements between CHARA and several institutions, our beam combination capabilities have been very significantly expanded beyond what could otherwise have been created by NSF funding and CHARA staff alone. The "CHARA Collaboration" includes groups (and their PIs) from l'Observatoire de Paris (Vincent Coudé du Foresto), University of Michigan (John Monnier), University of Sydney (Peter Tuthill), the Australian National University (Michael Ireland), l'Observatoire de la Côte d'Azur (Denis Mourard), and the University of Exeter (Stefan Kraus).

CHARA Classic $^{8}$ is a two-beam $J, H$ and $K$ open-air, beamsplitter-based system providing moderate-accuracy visibilities $(5 \%)$ at moderate sensitivity limits $(K=+8.5)$. Classic was our "first fringe" beam combiner and has been, until recently, our most productive science engine.

$\boldsymbol{C L I M B}^{10}$ the CLassic In Multiple Baselines beam combiner uses the pupil-plane implementation of Classic in the form of dual three-way beam combiners to obtain $J, H$, and $K$ visibilities and closure phases using two three-telescope sets. The first images based solely on CLIMB data were made of $\varepsilon$ Aur in $2011^{11}$. CLIMB can also be used as a fringe tracker for VEGA or PAVO.

$\boldsymbol{F L U O R}^{12}$ the Fiber Linked Unit for Optical Recombination is a two-beam, single-mode, fiber-based $K$-band beam combiner installed in 2003 as a result of our ongoing collaboration with the Paris group. FLUOR was the first beam 
combiner ever built to incorporate spatial filtering at the fiber inputs, and achieves our highest accuracy in $V^{2}( \pm 0.2 \%)$ at the expense of sensitivity $(K=+5.0)$. FLUOR recently underwent a major overhaul ${ }^{13}$ to increase throughput, add spectral resolution, and better integrate it into the CHARA Array environment.

MIRC $^{14}$, the Michigan InfraRed Combiner was funded by the NSF and the University of Michigan. MIRC permits amplitude and phase-closure imaging through six-telescope beam combination. Closure phases and amplitudes from four-telescope combination resulted in the publication of the first image ever of the photosphere of a main sequence star other than the Sun ${ }^{15}$. In 2017 MIRC will be upgraded to use a SELEX MOVPE SAPHIRA Array, funded through an ERC grant with PI Stefan Kraus. These state of the art arrays have demonstrated a noise level of 0.8 e-, allowing photon counting detection in the $H$-band. The upgraded beam combiner will be called MIRCX.

$\boldsymbol{V E G} \boldsymbol{A}^{16}$, the Visible spEctroGraph and polArimeter, is a four-beam, high spectral resolution $(\mathrm{R}=30,000)$, visible-light instrument installed at the CHARA Array in 2007- 2008. Operation of VEGA is now routine, and a beam combiner that will combine all six telescopes is now in the design phase, with a prototype already being tested on the sky ${ }^{17}$. VEGA represents the only visible-light, high-spatial and high-spectral resolution capability anywhere in the world.

$\boldsymbol{P A V O ^ { 1 8 }}$, the Precision Astronomical Visible Observation instrument is a 3-beam, low spectral resolution, visible-light beam combiner. PAVO has lower spectral resolution than VEGA but addresses the faintness limit by exploiting recent advances in optics and electro-optics.

MYSTIC, the Michigan Young STar Imager for CHARA, funded by NSF/ATI in 2015, is a new six-way NIR combiner being built by John Monnier's group in Michigan. MYSTIC is specifically designed to image planet-forming disks around YSOs. Like the MIRC upgrade, MYSTIC will take advantage of the new SELEX low noise detector technology. The optical core of MYSTIC will be a highly optimized integrated optics combiner made available through a strategic collaboration with the Institut de Planetologie et d'Astrophysique de Grenoble. The combination of MIRCX and MYSTIC using SELEX detectors, along with the CHARA AO program described below, will give the CHARA Array imaging capability simultaneously in $J, H$ and $K$-bands, with some spectral resolution and much greater sensitivity.

\subsection{Remote Operations}

The CHARA Array consortium has remote operations facilities in Atlanta, Sydney, Paris, Nice, and Michigan. Mobile laptop-based remote observing is now also available through a customized Linux distribution

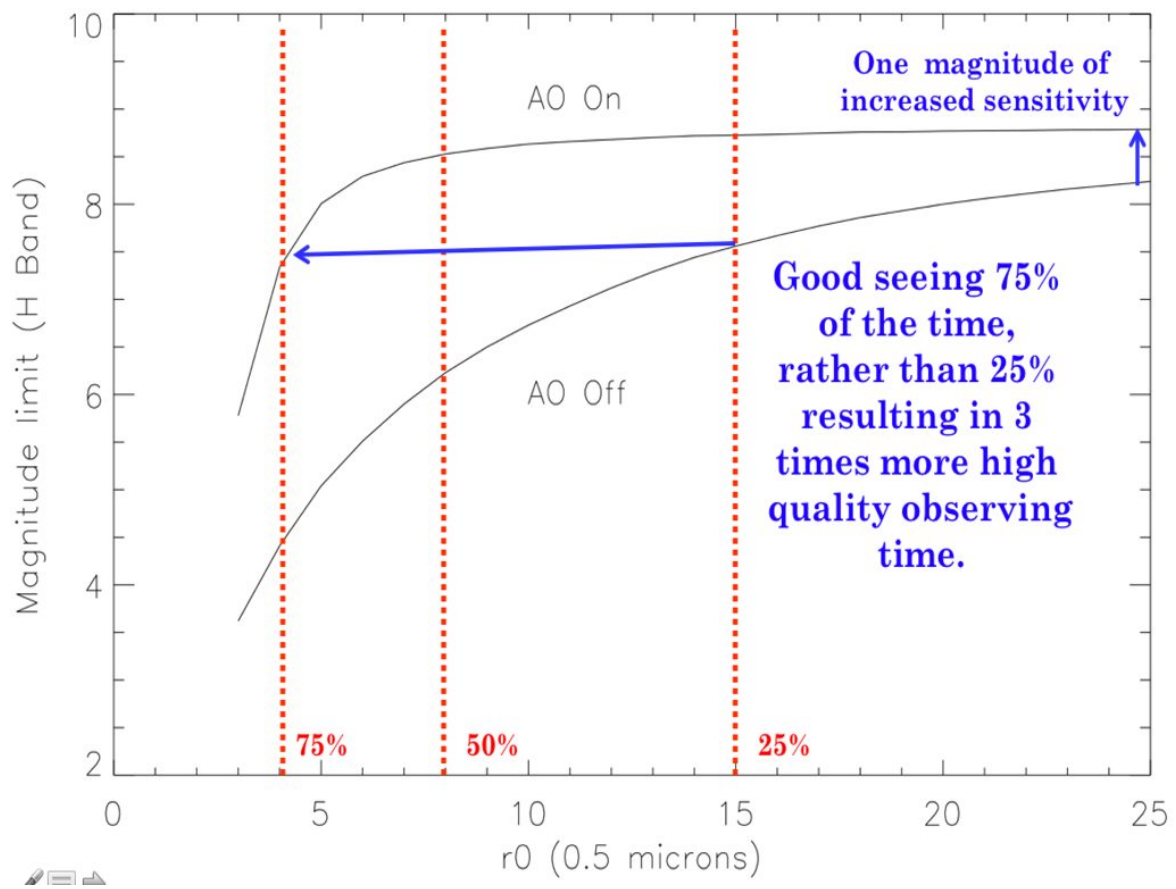

Figure 2. The magnitude limit of the CLASSIC beam combiner in the $H$-band as a function of Fried parameter in $\mathrm{cm}$ with, and without, the AO system. 


\section{THE CHARA ADAPTIVE OPTICS PROGRAM}

The CHARA Array Adaptive Optics (CHARA-AO) program ${ }^{19,20,21} \& 22$ has been split into two phases, primarily due to funding realities. CHARA-AO began in 2010 when Phase I of the program was funded by a $\$ 1.1 \mathrm{M}$ NSF/ATI grant. Phase I of our AO upgrade included wavefront sensors (WFS) at each telescope and a non-common path AO system for each telescope in the laboratory. Phase II, which includes large deformable mirrors (DM) for each telescope, was funded by NSF/MRI in 2015 and is now underway.

Figure 2 shows the modeled improvements provided by the full AO system at the CHARA Array (Che et al. 2013). With AO we will reach $H>8.5$ for $75 \%$ of the time, a threefold increase in high quality observing time, along with at least a one magnitude increase $50 \%$ of the time.

Up until now, the only interferometers able to measure fringes on objects fainter than $K>8$ have required 8-m class telescopes (KI, VLTI). These upgrades to the CHARA Array are responsive to the demands of the community: many competitive proposals for the CHARA public time focused on faint targets.
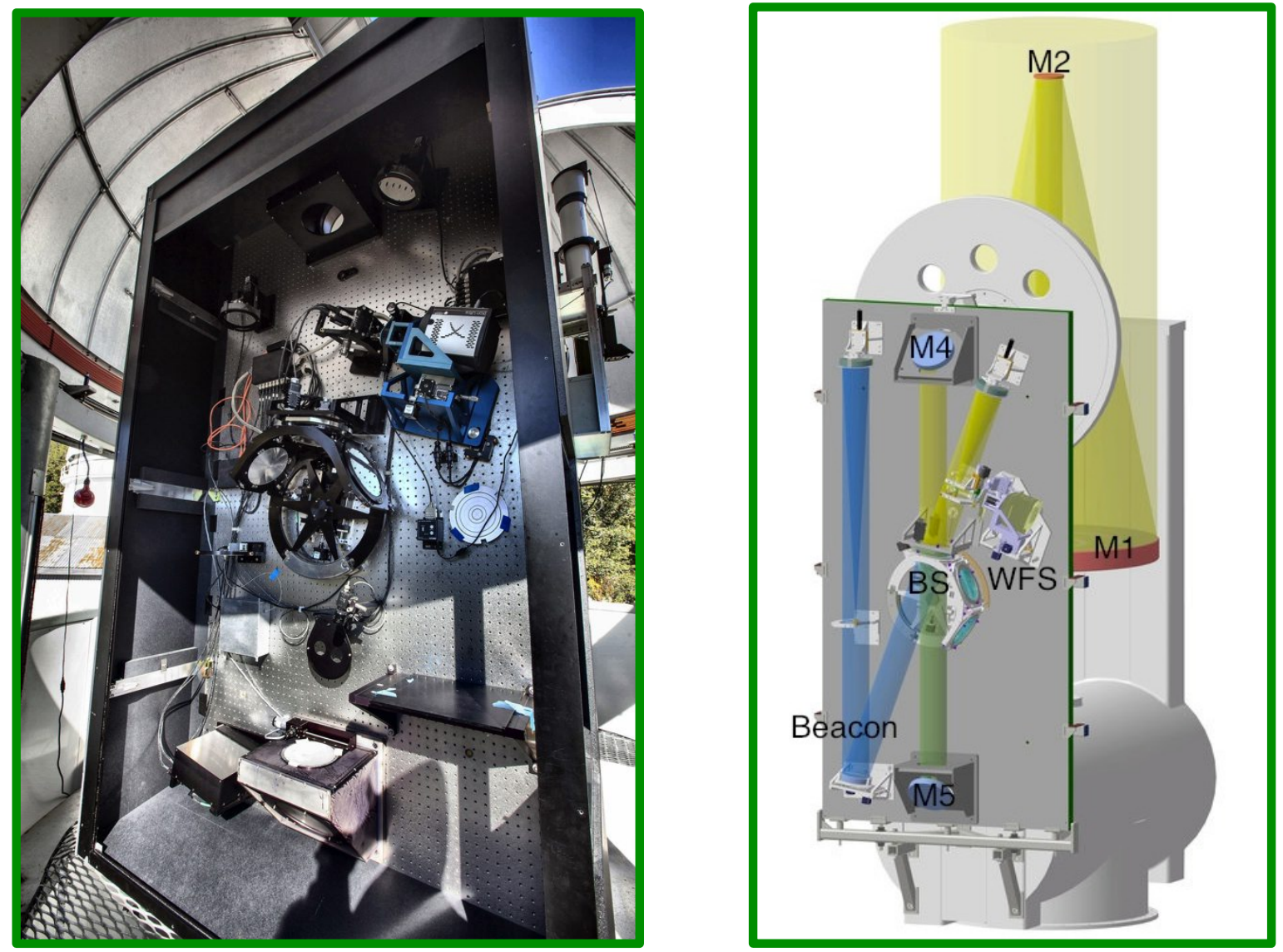

Figure 3. The CHARA-AO Phase-I components as installed on one of the CHARA Array telescopes.

\subsection{CHARA-AO Phase I}

The first phase of the CHARA-AO program is nearing completion. Large optical tables have been installed on the side of each telescope, as shown in Figure 3, that include a rotational platform to select one of three dichroic components, and a blue light beacon that can send light into both the local WFS, and into the laboratory for use by a second WFS and DM. Since there can be many hundreds of meters of optical path between the telescopes and the beam combiner, we have a significant non-common path issue at the CHARA Array, making the beacon and second AO system necessary. This LAB-AO system can also be used in closed loop on bright stars, since before we were funded to begin Phase-II the DM in the laboratory may have been the only one available for this purpose. Phase I of the CHARA-AO program will be completed in the fall of 2016. 


\subsection{CHARA-AO Phase II}

Phase-II of the CHARA-AO program has only just gotten underway, and primarily involves replacing the M4 mirrors with large deformable mirrors with 60-70 actuators. Since this mirror has an angle of incidence of 45 degrees, the actuator layout needs to be in an ellipse, rather than a circle, and the mirror is large as the beam size is $12.5 \mathrm{~cm}$. Thus, this must be a custom made device. A call for proposals went out late in 2015, and we received six viable proposals from a range of vendors. We were pleased to see such a response, as it was larger than we had expected, and somewhat surprised to see a rather small range in prices, with a standard deviation of only $10 \%$. Just before this meeting two vendors were selected as the primary and secondary sources and we are now in negotiation with the primary vendor. We hope to receive the first DM in the first quarter of 2017 and to be testing it on sky that summer.

\section{CHARA FUNDING NOW AND IN THE NEAR FUTURE}

The College of Arts and Sciences of Georgia State University, who provide the salaries of the senior personnel on the mountain, as well as equipment and supply funding, primarily fund the CHARA Array program. The remainder of the funding for personnel on the mountain is provided through an NSF/AAS grant on a five yearly basis. Current NSF funding is in place until late 2017 and we are now beginning preparation of the next NSF proposal due later in 2016. Other large hardware development funds are based on proposals to the NSF/ATI, NSF/MRI and ERC, for example the MIRCX, MYSTIC, and CHARA-AO programs described above. The CHARA Array is a PI led University based research program, and like many others of its kind, we need to be continually looking for ways to improve the system, leverage these improvements for science, and use this as a means to seek new funding for new improvements.

One way we have been pursuing increasing the scientific output of the Array is to open up more time for the community through the NOAO. For six years we have been offering small amounts of time through the NOAO time allocation process, and this has been over subscribed by factors of 3-5 each year. We have recently proposed to expand this program through the NSF/MSIP mechanism. The MSIP (Mid-Scale Innovations Program in Astronomical Sciences) program is a response to the previous decadal report that stated that the NSF needs to direct more funding to mid-scale $(\$ 4-\$ 40 \mathrm{M})$ programs, including new instruments, access to existing instruments, and access to existing data archives. In this program we would open up 50-75 nights per year to the world wide astronomical community through the NOAO/TAC. The NOAO Director has kindly agreed to continue processing proposals for the greatly expanded access that MSIP will enable. NOAO standards and practices for proposal solicitation and review, and for proprietary periods, will be followed and CHARA's only TAC involvement will be to comment on the technical feasibility of proposals. CHARA staff and consortium teams will not compete for MSIP time and there will be no reserved science topics.

As a second part of this MSIP program, we plan to create a CHARA data archive administered through the Jean-Marie Mariotti Center in Grenoble (JMMC) funded by the CNRS in France. This work has already begun, with all CLASSIC, CLIMB, FLUOR and VEGA level one data already available at the JMMC webpage. Like the open NOAO access time, it is impossible for us to predict exactly what use the community will make of the CHARA Array data archive, but like all astronomical data, it is equally unlikely that all possible astronomical insight will be derived from these data immediately after they have been obtained.

The final part of the MSIP program involves community development. Our contention is that the U.S. community lags behind the European community in exploitation of optical interferometry owing to lack of access and limited familiarity with the technique. We hope to address both factors by offering access and support, and sponsoring 10-12 Community Development Workshops in strategic locations around the U.S. modeled after the very successful program undertaken at NRAO to develop a user community for ALMA, which opened a new observational methodology just as we now wish to do. The ALMA experience has shown that a series of localized workshops is a very effective way to build a user community to take advantage of a very new capability.

In order to support this program we have asked for support for 5 new positions on the mountain, funding for improvements to Array infrastructure, and travel funds to enable visiting scientists who obtain time on the Array to come to the mountain during their observing runs. We hope to add a Visitor Support Scientist to oversee recruitment workshops and assist new observers, answer questions in the proposal process, and ensure that the final data products fulfill observational goals. A full-time Data Scientist will be required to oversee data pipelines, assist in data reduction, and to work with JMMC on the Data Archive. A third Array Operator was requested to staff fully this vital role with professional operators. Finally a Machinist/Technician and a Technician to assist the CHARA Site Manager in the myriad tasks associated with maintaining our six-telescopes, our delay line and beam combination facility and our other 
buildings. For infrastructure we have identified components in various subsystems that make the Array vulnerable to single-point failure situations in which one telescope or even all six can be disabled. CHARA has had insufficient budget resources to maintain a proper inventory of spares for such items, and so we requested funds in the first two years to secure critical spares as well to as upgrade aging and dated control systems.

At the time of writing, we have not yet heard the outcome of this proposal. Should it be successful, we hope to start hiring the new staff late in 2016.

\section{CONCLUSION}

This is necessarily a very short summary of developments over the last two years, and of our hopes for the next few years. We believe that the CHARA Array needs to evolve from a PI based instrument and move towards becoming a more general-purpose resource for the Astronomical community at large. Contemporary astronomy has drifted away from narrow specialization so that few would today identify themselves strictly as a "spectroscopist" or a "photometrist," and most seek to explore their scientific interests through a variety of techniques operating in a range of spectral bandpasses. Our goal is, along with our colleagues at other facilities, to help establish Long Baseline Optical Interferometry as another powerful and accessible tool available within the U.S. and international observational ensemble.

\section{REFERENCES}

[1] H. A. McAlister, T. A. ten Brummelaar, and S. T. Ridgway, "Making the CHARA Array, Part I: founding CHARA, the audacity of hope," Proc. SPIE 9141 (2014).

[2] S. T. Ridgway, T. A. ten Brummelaar, and H. A. McAlister, "Making the CHARA Array, Part II: project management: 15 years on thin ice," Proc. SPIE 9141 (2014).

[3] T. A. ten Brummelaar, H. A. McAlister, and S. Ridgway, "Making the CHARA Array, Part III: engineering decisions. To build or not to build," Proc. SPIE 9141 (2014).

[4] T. A. ten Brummelaar, H. A. McAlister, S. T. Ridgway, D. R. Gies, J. Sturmann, L. Sturmann, N. H. Turner, G. H. Schaefer, C. D. Farrington, and P. J. Goldfinger, "An update on the CHARA Array," Proc. SPIE 7734 (2010).

[5] T. A. ten Brummelaar, H. A. McAlister, S. Ridgway, D. R. Gies, J. Sturmann, L. Sturmann, N. H. Turner, A. Merand, R. Thompson, C. D. Farrington, and P. J. Goldfinger, "An update on the CHARA array," Proc. SPIE 7013 (2008).

[6] T. A. ten Brummelaar, H. A. McAlister, S. T. Ridgway, N. H. Turner, L. Sturmann, J. Sturmann, W. G. Bagnuolo, Jr., and M. A. Shure, "An Update of the CHARA Array.” Proc. SPIE 4838, 69-78, (2003).

[7] T. A. ten Brummelaar, W. G. Bagnuolo, H. A. McAlister, S. T. Ridgway, L. Sturmann, J. Sturmann, and N. H. Turner, "Technical update of the CHARA array" Proc. SPIE 4006, 564-573 (2000).

[8] T. A. ten Brummelaar, H. A. McAlister, S. T. Ridgway, W. G. Bagnuolo, Jr., N. H. Turner, L. Sturmann, J. Sturmann, D. H. Berger, C. E. Ogden, R. Cadman, W. I. Hartkopf, C. H. Hopper, and M. A. Shure., "First Results from the CHARA Array. II. A Description of the Instrument." ApJ, 628, 453-465, (2005).

[9] Berger, D. H., "Longitudinal Dispersion Compensation for a Long Baseline Optical Interferometer," PASP, 116, 390-390. (2004)

[10] ten Brummelaar, T. A., Sturmann, J., Ridgway, S. T., Sturmann, L., Turner, N. H., McAlister, H. A., Farrington, C. D., Beckmann, U., Weigelt, G., and Shure, M, "The Classic/climb Beam Combiner at the CHARA Array.” Journal of Astronomical Instrumentation, 2, 40004 (2013)

[11] Kloppenborg, B. K., Stencel, R. E., Monnier, J. D., Schaefer, G. H., Baron, F., Tycner, C., Zavala, R. T., Hutter, D., Zhao, M., Che, X., ten Brummelaar, T. A., Farrington, C. D., Parks, R., McAlister, H. A., Sturmann, J., Sturmann, L., Sallave- Goldfinger, P. J., Turner, N., Pedretti, E., and Thureau, N, "Interferometry of a Aurigae: Characterization of the Asymmetric Eclipsing Disk," ApJS, 220, 14 (2015)

[12] Coude du Foresto, V., Borde, P. J., Merand, A., Baudouin, C., Remond, A., Perrin, G. S., Ridgway, S. T., ten Brummelaar, T. A., and McAlister, H. A., "FLUOR fibered beam combiner at the CHARA array," Proc. SPIE, 4838, 280-285. (2003)

[13] Scott, N. J., Millan-Gabet, R., Lhome, E., ten Brummelaar, T. A., Coude Du Foresto, V., Sturmann, J., and Sturmann, L., "Jouvence of Fluor: Upgrades of a Fiber Beam Combiner at the CHARA Array. Journal of Astronomical Instrumentation, 2, 40005. (2013) 
[14] Monnier, J. D., Pedretti, E., Thureau, N., Berger, J.-P., Millan-Gabet, R., ten Brum- melaar, T. A., McAlister, H., Sturmann, J., Sturmann, L., Muirhead, P., Tannirkulam, A., Webster, S., and Zhao, M., "Michigan Infrared Combiner (MIRC): commissioning results at the CHARA Array," Proc SPIE 6268, 1P (2006)

[15] Monnier, J. D., Zhao, M., Pedretti, E., Thureau, N., Ireland, M., Muirhead, P., Berger, J.-P., Millan-Gabet, R., Van Belle, G., ten Brummelaar, T. A., McAlister, H., Ridgway, S., Turner, N., Sturmann, L., Sturmann, J., and Berger, D. "Imaging the Surface of Altair," Science, 317, 342 (2007).

[16] Mourard, D., Clausse, J. M., Marcotto, A., Perraut, K., Tallon-Bosc, I., Berio, P., Blazit, A., Bonneau, D., Bosio, S., Bresson, Y., Chesneau, O., Delaa, O., Henault, F., Hughes, Y., Lagarde, S., Merlin, G., Roussel, A., Spang, A., Stee, P., Tallon, M., Antonelli, P., Foy, R., Kervella, P., Petrov, R., Thiebaut, E., Vakili, F., McAlister, H., ten Brummelaar, T. A., Sturmann, J., Sturmann, L., Turner, N., Farrington, C., and Goldfinger, P. J., "VEGA: Visible spEctroGraph and polArimeter for the CHARA array: principle and performance," A\&A, 508, 1073-1083 (2009)

[17] Berio, P., Bresson, Y., Clausse, J. M., Mourard, D., Dejonghe, J., Duthu, A., La- garde, S., Meilland, A., Perraut, K., Tallon-Bosc, I., Nardetto, N., Spang, A., Bailet, C., Marcotto, A., Chesneau, O., Stee, P., Feautrier, P., Balard, P., and Gach, J. L., "Long baseline interferometry in the visible: the FRIEND project," Proc SPIE 9146, 914616. (2014)

[18] Ireland, M. J., Merand, A., ten Brummelaar, T. A., Tuthill, P. G., Schaefer, G. H., Turner, N. H., Sturmann, J., Sturmann, L., and McAlister, H. A., "Sensitive visible interferometry with PAVO. In Optical and Infrared Interferometry," Proc. SPIE 7013, 701324, (2008)

[19] X. Che, L. Sturmann, J. D. Monnier, T. A. Ten Brummelaar, J. Sturmann, S. T. Ridgway, M. J. Ireland, N. H. Turner, and H. A. McAlister, "Optical and Mechanical Design of the CHARA Array Adaptive Optics," Journal of Astronomical Instrumentation, 2, 40007 (2013)

[20] X. Che, L. Sturmann, J. D. Monnier, T. A. ten Brummelaar, J. Sturmann, S. T. Ridgway, M. J. Ireland, N. H. Turner, and H. A. McAlister, "The CHARA array adaptive optics I: common-path optical and mechanical design, and preliminary on- sky results," Proc. SPIE 9148, 30 (2014)

[21] T. A. ten Brummelaar, X. Che, H. McAlister, M. Ireland, J. Monnier, D. Mourard, S. Ridgway, J. Sturmann, L. Sturmann, N. Turner, and P. Tuthill, "CHARA array adaptive optics II: non-common-path correction and downstream optics," Proc. SPIE 9148, 4, (2014)

[22] T. A. ten Brummelaar, L. Sturmann, J. Sturmann, S. T. Ridgway, J. D. Monnier, M. J. Ireland, X. Che, H. A. McAlister, N. H. Turner, and P. G. Tuthill, “Adaptive optics for the CHARA array,” Proc. SPIE 8447, 3, (2012) 